\title{
Plasma deoxysphingolipids: a novel class of biomarkers for the metabolic syndrome?
}

\author{
A. Othman • M. F. Rütti • D. Ernst • C. H. Saely • \\ P. Rein • H. Drexel • C. Porretta-Serapiglia • G. Lauria • \\ R. Bianchi • A. von Eckardstein • T. Hornemann
}

Received: 12 July 2011 / Accepted: 10 October 2011 /Published online: 29 November 2011

(C) Springer-Verlag 2011

\begin{abstract}
Aims/hypothesis Sphingolipid synthesis is typically initiated by the conjugation of L-serine and palmitoyl-CoA, a reaction catalysed by serine palmitoyltransferase (SPT). SPT can also metabolise other acyl-CoAs $\left(\mathrm{C}_{12}\right.$ to $\left.\mathrm{C}_{18}\right)$ and other amino acids such as L-alanine and glycine, giving rise to a spectrum of atypical sphingolipids. Here, we aimed to identify changes in plasma levels of these atypical sphingolipids to explore their potential as biomarkers in the metabolic syndrome and diabetes.
\end{abstract}

A. Othman and M. F. Rütti contributed equally to this study.

The nomenclature used in this paper generally conforms to the recommendations of the IUPAC-IUB Commission of Biochemical Nomenclature [1] (see Appendix).

Electronic supplementary material The online version of this article (doi:10.1007/s00125-011-2384-1) contains peer-reviewed but unedited supplementary material, which is available to authorised users.

A. Othman • M. F. Rütti • D. Ernst • A. von Eckardstein •

T. Hornemann $(\bowtie)$

Institute for Clinical Chemistry, University Hospital Zurich,

Rämistrasse 100,

8091 Zurich, Switzerland

e-mail: thorsten.hornemann@usz.ch

A. Othman · D. Ernst • A. von Eckardstein · T. Hornemann Centre for Integrative Human Physiology, University of Zurich, Zurich, Switzerland

\section{F. Rütti}

Division of Internal Medicine, Hospital Wil, Wil, Switzerland

A. Othman · A. von Eckardstein · T. Hornemann Competence Centre for Systems Physiology and Metabolic Diseases,

Zurich, Switzerland
Methods We compared the plasma profiles of ten sphingoid bases in healthy individuals with those of patients with the metabolic syndrome but not diabetes, and diabetic patients ( $n=25$ per group). The results were verified in a streptozotocin (STZ) rat model. Univariate and multivariate statistical analyses were used.

Results Deoxysphingolipids (dSLs) were significantly elevated $\left(p=5 \times 10^{-6}\right)$ in patients with the metabolic syndrome $(0.11 \pm 0.04 \mu \mathrm{mol} / \mathrm{l})$ compared with controls $(0.06 \pm 0.02 \mu \mathrm{mol} / \mathrm{l})$ but did not differ between the metabolic

C. H. Saely $\cdot$ P. Rein $\cdot$ H. Drexel

Vorarlberg Institute for Vascular Investigation and Treatment (VIVIT),

Feldkirch, Austria

C. H. Saely $\cdot$ P. Rein $\cdot$ H. Drexel

Department of Medicine and Cardiology,

Academic Teaching Hospital Feldkirch,

Feldkirch, Austria

C. H. Saely $\cdot$ P. Rein $\cdot$ H. Drexel

Private University of the Principality of Liechtenstein,

Triesen, Liechtenstein

H. Drexel

Drexel University College of Medicine,

Philadelphia, PA, USA

C. Porretta-Serapiglia $\cdot$ G. Lauria $\cdot$ R. Bianchi

Carlo Besta Foundation IRCCS National Neurological Institute, Milan, Italy 
syndrome and diabetes groups. Levels of $\mathrm{C}_{16}$-sphingosinebased sphingolipids were significantly lowered in diabetic patients but not in patients with the metabolic syndrome but without diabetes $(p=0.008)$. Significantly elevated dSL levels were also found in the plasma and liver of STZ rats. A principal component analysis revealed a similar or even closer association of dSLs with diabetes and the metabolic syndrome in comparison with the established biomarkers. Conclusions/interpretation We showed that dSLs are significantly elevated in patients with type 2 diabetes mellitus and non-diabetic metabolic syndrome compared with healthy controls. They may, therefore, be useful novel biomarkers to improve risk prediction and therapy monitoring in these patients.

Keywords Atypical sphingolipids · Biomarker .

Deoxysphingolipids $\cdot$ Metabolic syndrome $\cdot$ Type 2 diabetes mellitus

$\begin{array}{ll}\text { Abbreviations } \\ \text { ALT } & \text { Alanine aminotransferase } \\ \text { doxSA } & \text { Deoxysphinganine } \\ \text { doxSO } & \begin{array}{l}\text { Deoxysphingosine } \\ \text { dSL }\end{array} \\ \text { Deoxysphingolipid } \\ \text { DPN } & \text { Diabetic peripheral neuropathy } \\ \text { HSAN1 } & \begin{array}{l}\text { Hereditary sensory and autonomic } \\ \text { neuropathy type 1 }\end{array} \\ \text { OPLS-DA } & \text { Orthogonal partial least square-discriminant } \\ & \text { analysis } \\ \text { PCA } & \text { Principal component analysis } \\ \text { ROC } & \text { Receiver operating characteristic } \\ \text { SA } & \text { Sphinganine } \\ \text { SO } & \text { Sphingosine } \\ \text { SPT } & \text { Serine palmitoyltransferase } \\ \text { STZ } & \text { Streptozotocin } \\ \text { VIP } & \text { Variable importance for the projection }\end{array}$

\section{Introduction}

The pathogenesis of diabetes mellitus type 2 is characterised by the development of insulin resistance, frequently because of excess body fat, that is initially overcome by increased insulin secretion and-after several years - a subsequent decrease in the functional pancreatic beta cell mass that can no longer compensate for insulin resistance [2-4]. Insulin resistance or the compensating hyperinsulinaemia leads to the manifestation of a cluster of risk factors, including hyperglycaemia, hypertriacylglycerolaemia, low plasma levels of HDL-cholesterol and arterial hypertension, that has been termed the metabolic syndrome. Although much progress [5] has been made in the understanding of the mutual relationships between obesity, insulin resistance, type 2 diabetes and atherosclerosis, the complete picture remains elusive. In the last years the carbohydrate-centred view of the pathogenesis of diabetes has widened to include different classes of lipids [5-7] and inflammatory factors [8]. Mounting evidence suggests that sphingolipids play a role in the pathogenesis of insulin resistance and diabetes [9-11]. In particular, ceramides have been suggested to be important in the development of insulin resistance. For instance, inhibition of ceramide synthesis restored insulin sensitivity in palmitate-induced insulin resistance in mice [12].

Sphingolipids comprise a heterogeneous class of lipids that are derived from the aliphatic amino-alcohol sphingosine, which is commonly formed from the precursors L-serine and palmitoyl-CoA (electronic supplementary material [ESM] Fig. 1). This reaction is catalysed by the enzyme serine palmitoyltransferase (SPT). In addition to these substrates, SPT metabolises other acyl-CoAs with carbon chain lengths in the range $\mathrm{C}_{12}$ to $\mathrm{C}_{18}$ [13]. Moreover, SPT also has flexibility in the use of other amino acid substrates such as L-alanine and glycine $[14,15]$, which generate an atypical category of 1-deoxysphingoid bases. Whereas the conjugation of alanine forms deoxysphinganine (doxSA), the use of glycine results in the formation of deoxymethylsphinganine. These two metabolites can be $N$-acylated to form deoxysphingolipids (dSLs), but cannot be further metabolised to complex sphingolipids because of the missing $\mathrm{C}_{1}$-hydroxyl group [16]. Consequently, these metabolites cannot be degraded by the canonical sphingolipid degradation pathway, which requires the formation of sphingosine-1phosphate as a catabolic intermediate. Whereas dSLs occur ubiquitously at low levels in human plasma, levels are greatly increased in hereditary sensory and autonomic neuropathy type 1 (HSAN1; OMIM162400), an inherited sensory neuropathy caused by missense mutations in SPT [15].

Physiologically, de novo sphingolipid synthesis represents a metabolic cross point that interconnects lipid, amino acid and thereby indirectly also carbohydrate metabolism. Fluctuations in fatty acid and thereby acyl-CoA concentrations are reflected in altered carbon-chain compositions of the sphingoid bases [12, 17]. Another node connects serine and alanine metabolism to the sphingolipid metabolism. Serine is formed from 3-phosphoglycerate whereas alanine can be converted from and to pyruvate through alanine aminotransferase (ALT) in a reversible transaminase reaction. Hence, the precursors for both amino acids are generated during glycolysis and thereby provide a functional link between sphingolipid and carbohydrate metabolism. In this respect, it is noteworthy that alanine is the major gluconeogenic amino acid, with important roles in obesity and diabetes $[18,19]$.

In light of these metabolic connections and the increasing evidence that certain sphingolipid molecules have roles in the pathogenesis of insulin resistance and diabetes, we 
Table 1 Baseline characteristics and results

\begin{tabular}{|c|c|c|c|c|c|c|}
\hline \multirow[t]{2}{*}{ Characteristic } & \multirow[t]{2}{*}{ Control $(n=25)$} & \multirow{2}{*}{$\begin{array}{l}\text { Metabolic syndrome } \\
(n=25)\end{array}$} & \multirow[t]{2}{*}{ Diabetes $(n=25)$} & \multirow[t]{2}{*}{ Kruskal-Wallis $p$ value } & \multicolumn{2}{|c|}{ Mann-Whitney $U p$ value } \\
\hline & & & & & C vs MetS & MetS vs D \\
\hline Age & $61.19 \pm 4.28$ & $62.13 \pm 5.02$ & $61.56 \pm 4.64$ & 0.771 & 0.50 & 0.567 \\
\hline Female $(\%)$ & 40 & 40 & 40 & 1.000 & & \\
\hline Smoking (\%) & 64 & 56 & 60 & 0.846 & & \\
\hline $\mathrm{Wcf}(\mathrm{cm})$ & $98.24 \pm 12.10$ & $105.84 \pm 8.23$ & $110.80 \pm 11.97$ & $3.70 \times 10^{-4}$ & 0.004 & 0.051 \\
\hline BMI & $28.19 \pm 4.49$ & $30.13 \pm 3.01$ & $32.21 \pm 4.83$ & 0.006 & 0.05 & 0.09 \\
\hline Fasting glucose $(\mathrm{mmol} / \mathrm{l})$ & $5.26 \pm 0.56$ & $5.64 \pm 0.57$ & $9.59 \pm 4.16$ & $1.94 \times 10^{-9}$ & 0.03 & $4.29 \times 10^{-7}$ \\
\hline $\mathrm{HbA}_{1 \mathrm{c}}(\%)$ & $5.60 \pm 0.33$ & $5.76 \pm 0.34$ & $7.79 \pm 2.04$ & $4.71 \times 10^{-10}$ & 0.17 & $9.52 \times 10^{-8}$ \\
\hline $\mathrm{HbA}_{1 \mathrm{c}}(\mathrm{mmol} / \mathrm{l})$ & 37.71 & 39.45 & 61.64 & & & \\
\hline Cholesterol (mmol/l) & $5.26 \pm 1.24$ & $4.99 \pm 1.30$ & $4.98 \pm 1.44$ & 0.777 & 0.49 & 0.861 \\
\hline LDL-cholesterol (mmol/1) & $3.35 \pm 0.96$ & $3.45 \pm 1.00$ & $3.16 \pm 1.22$ & 0.449 & 0.52 & 0.269 \\
\hline HDL-cholesterol (mmol/l) & $1.80 \pm 0.69$ & $1.15 \pm 0.21$ & $1.31 \pm 0.32$ & $1.60 \times 10^{-5}$ & $2.37 \times 10^{-6}$ & 0.132 \\
\hline TG $(\mathrm{mmol} / \mathrm{l})$ & $1.10 \pm 0.37$ & $2.51 \pm 0.83$ & $2.21 \pm 1.47$ & $1.96 \times 10^{-7}$ & $1.38 \times 10^{-8}$ & 0.067 \\
\hline SBP $(\mathrm{mmHg})$ & $126.80 \pm 12.47$ & $133.84 \pm 15.23$ & $135.52 \pm 15.92$ & 0.081 & 0.12 & 0.44 \\
\hline DBP $(\mathrm{mmHg})$ & $78.80 \pm 8.50$ & $83.92 \pm 9.41$ & $82.24 \pm 8.99$ & 0.161 & 0.06 & 0.565 \\
\hline AST & $28.56 \pm 8.60$ & $30.84 \pm 24.10$ & $25.84 \pm 8.43$ & 0.388 & 0.38 & 0.662 \\
\hline ALT & $29.92 \pm 15.71$ & $34.76 \pm 24.54$ & $31.68 \pm 13.25$ & 0.650 & 0.35 & 0.907 \\
\hline Creatinine & $0.84 \pm 0.18$ & $0.92 \pm 0.21$ & $0.81 \pm 0.23$ & 0.144 & 0.20 & 0.06 \\
\hline GFR & $100.65 \pm 11.53$ & $95.26 \pm 15.30$ & $100.77 \pm 15.68$ & 0.316 & 0.22 & 0.184 \\
\hline $\mathrm{C}_{16} \mathrm{SO}(\mu \mathrm{mol} / \mathrm{l})$ & $8.31 \pm 4.49$ & $8.38 \pm 2.25$ & $6.37 \pm 2.82$ & 0.041 & 0.55 & 0.008 \\
\hline $\mathrm{C}_{16} \mathrm{SA}(\mu \mathrm{mol} / \mathrm{l})$ & $0.23 \pm 0.13$ & $0.23 \pm 0.10$ & $0.26 \pm 0.14$ & 0.749 & 0.84 & 0.491 \\
\hline $\mathrm{C}_{17} \mathrm{SO}(\mu \mathrm{mol} / \mathrm{l})$ & $4.66 \pm 2.42$ & $4.02 \pm 1.10$ & $3.71 \pm 1.48$ & 0.387 & 0.64 & 0.29 \\
\hline $\mathrm{C}_{17} \mathrm{SA}(\mu \mathrm{mol} / \mathrm{l})$ & $0.15 \pm 0.07$ & $0.14 \pm 0.05$ & $0.14 \pm 0.05$ & 0.960 & 0.82 & 0.961 \\
\hline $\mathrm{SO}(\mu \mathrm{mol} / \mathrm{l})$ & $88.83 \pm 27.23$ & $81.66 \pm 21.28$ & $81.54 \pm 23.89$ & 0.622 & 0.34 & 0.793 \\
\hline $\mathrm{SA}(\mu \mathrm{mol} / 1)$ & $2.17 \pm 0.9$ & $2.20 \pm 0.77$ & $2.57 \pm 1.14$ & 0.473 & 0.82 & 0.299 \\
\hline $\mathrm{C}_{20} \mathrm{SO}(\mu \mathrm{mol} / \mathrm{l})$ & $0.20 \pm 0.08$ & $0.19 \pm 0.06$ & $0.19 \pm 0.05$ & 0.727 & 0.46 & 0.839 \\
\hline $\operatorname{doxSO}(\mu \mathrm{mol} / \mathrm{l})$ & $0.15 \pm 0.09$ & $0.23 \pm 0.09$ & $0.24 \pm 0.13$ & $2.14 \times 10^{-4}$ & $3 \times 10^{-5}$ & 0.839 \\
\hline $\operatorname{doxSA}(\mu \mathrm{mol} / \mathrm{l})$ & $0.06 \pm 0.02$ & $0.11 \pm 0.04$ & $0.12 \pm 0.05$ & $3.92 \times 10^{-6}$ & $5 \times 10^{-6}$ & 0.808 \\
\hline $\mathrm{C}_{18} \mathrm{SA}$-diene $(\mu \mathrm{mol} / \mathrm{l})$ & $20.79 \pm 8.17$ & $18.27 \pm 5.74$ & $16.42 \pm 6.80$ & 0.148 & 0.36 & 0.273 \\
\hline
\end{tabular}

Values are expressed as means $\pm \mathrm{SD}$ or percentages for scale or categorical variables, respectively

$p$ values were calculated with Kruskal-Wallis test for all three group, and with the Mann-Whitney $U$ test for two group comparisons; after Bonferroni correction a $p$ value of 0.002 corresponded to a significance level of 0.05 . For the categorical variable, asymptotic two-sided $p$ values for $\chi^{2}$ are shown

AST, aspartate aminotransferase; C, controls; D, diabetic patient group; DBP, diastolic BP; MetS, metabolic syndrome group; SBP, systolic BP; TG, triacylglycerol; Wcf, waist circumference

compared the plasma sphingoid base profiles of healthy humans with those of patients with the metabolic syndrome but not diabetes and of patients with type 2 diabetes mellitus. The observed differences were further confirmed in an animal model of diabetes.

\section{Methods}

\section{Patients}

A sex- and age-matched nested cohort of 25 patients with non-diabetic metabolic syndrome, 25 patients with the metabolic syndrome and type 2 diabetes mellitus, and 25 controls without diabetes or the metabolic syndrome was selected from a previously described study cohort [20] of the Vorarlberg Institute for Vascular Investigation and Treatment (VIVIT; Feldkirch, Austria). The samples were derived from a larger cohort of unselected white patients undergoing coronary angiography for the evaluation of suspected coronary artery disease. Only patients with negative angiographic results were enrolled in this study. The age range was 55-69 years. The angiographers were not aware of plasma sphingolipid levels. The study was approved by the Ethics Committee of the University of Innsbruck and all participants gave written informed consent. 
According to National Cholesterol Education ProgramAdult Treatment Panel III criteria, non-diabetic metabolic syndrome was diagnosed if three or more of the following criteria were fulfilled: waist circumference $>102 \mathrm{~cm}$ in men or $>88 \mathrm{~cm}$ in women; triacylglycerols $\geq 1.7 \mathrm{mmol} / 1$ $(150 \mathrm{mg} / \mathrm{dl})$; HDL-cholesterol $<1.0 \mathrm{mmol} / 1(40 \mathrm{mg} / \mathrm{dl})$ in men or $<1.3 \mathrm{mmol} / \mathrm{l}(50 \mathrm{mg} / \mathrm{dl})$ in women; blood pressure $\geq 130 / \geq 85 \mathrm{mmHg}$; and fasting glucose $\geq 6.1 \mathrm{mmol} / 1(110 \mathrm{mg} / \mathrm{dl})$ but $<7 \mathrm{mmol} / \mathrm{l}$. Type 2 diabetes mellitus was diagnosed by either fasting glucose levels $\geq 7 \mathrm{mmol} / 1(126 \mathrm{mg} / \mathrm{dl})$, or plasma glucose levels $\geq 11.1 \mathrm{mmol} / 1(200 \mathrm{mg} / \mathrm{dl}) 2 \mathrm{~h}$ after an oral challenge with $75 \mathrm{~g}$ glucose, or previously diagnosed diabetes. Controls were defined by the absence of both non-diabetic metabolic syndrome and type 2 diabetes mellitus

\section{Clinical chemistry}

Venous blood samples were collected after an overnight fast of at least $12 \mathrm{~h}$ before angiography was performed, and laboratory measurements were performed from fresh serum samples. The serum levels of triacylglycerols, total cholesterol and HDL-cholesterol were determined by using enzymatic hydrolysis and precipitation techniques (triacylglycerol glycerol phosphate oxidase- $p$-aminophenazone (GPO-PAP), cholesterol oxidase phenol 4-aminoantipyrine peroxidase (CHOD/PAP), QuantolipLDL, QuantolipHDL; Roche, Basel, Switzerland) on a Hitachi-Analyzer 717 or 911. Level of $\mathrm{HbA}_{1 \mathrm{c}}$ was determined by high-performance liquid chromatography (LC) on a Menarini-Arkray KDK HA 8140 (Arkray KDK, Kyoto, Japan). Clinical chemistry variables were measured on a Hitachi 717 or 911 system (Roche).

\section{Animal model}

Male Sprague-Dawley rats (180-200 g, Charles River, Calco, Italy) were housed in pairs. Animal room temperature and relative humidity were set at $22 \pm 2^{\circ} \mathrm{C}$ and $55 \pm$ $10 \%$, respectively. Artificial lighting provided a $12 \mathrm{~h}$ lightdark cycle (07:00-19:00 hours). The animals had free access to diet and water. Diabetes was induced in rats fasted overnight by a single i.p. injection of $60 \mathrm{mg} / \mathrm{kg}$ of streptozotocin (STZ) dissolved in sodium citrate buffer $(\mathrm{pH}$ 4.5). The control rats were injected with vehicle. Hyperglycaemia was confirmed by measuring glycosuria $72 \mathrm{~h}$ after STZ injection, using Keto-Diabur test 5000 strips (Roche Diagnostics, Spa, Italy). Only animals with glycosuria $>5 \%$ were classified as diabetic and included in the study. Body weight and blood glucose concentration, determined by tail bleeding using strips (Ascensia Elite; Bayer, Basel, Switzerland), were measured weekly. Immediately after killing the animals, liver and muscle (gastroc- nemius) were carefully dissected out and immediately frozen in liquid $\mathrm{N}_{2}$.

Quantification of sphingoid bases

The lipids were analysed as described before [15, 21]. Tissue samples were homogenised in lysis buffer (25 mmol/l HEPES pH 8, 0.2\% Triton X-100 [vol./vol.]) using a Precellys 24 tissue homogenizer (Bertin Technologies, Montigny-le-Bretonneux, France). Briefly, $100 \mu$ plasma or tissue homogenate containing $80 \mu \mathrm{g}$ extracted protein was added to $1 \mathrm{ml}$ extraction buffer $(2: 1)$ methanol-KOH/ chloroform and spiked with 200 pmol internal standard $\mathrm{C}_{20}$-sphinganine $\left(\mathrm{C}_{20} \mathrm{SA}\right.$; Avanti Polar Lipids, Alabaster, AL, USA).

The extracted dried lipids were acid hydrolysed using methanolic $\mathrm{HCl}(1 \mathrm{~mol} / \mathrm{l} \mathrm{HCl} / 10 \mathrm{~mol} / 1$ water in methanol) and heat $\left(65^{\circ} \mathrm{C}, 16 \mathrm{~h}\right)$ to release the sphingoid base backbones. This was followed by a second extraction. LC/ MS analysis was performed as described earlier [15]. Lipids were separated on a $\mathrm{C}_{18}$ column (Uptispere $120 \AA, 5 \mu \mathrm{m}$, $125 \times 2 \mathrm{~mm}$; Interchim, Montluçon, France) and analysed by an MS detector (LCQ, Thermo, Wohlen, Switzerland). The samples were measured as singletons for each participant. Inter- and intra-assay CVs were between $5 \%$ and $20 \%$ for each sphingoid base.

The sphingoid bases analysed included $\mathrm{C}_{16} \mathrm{SO}, \mathrm{C}_{16} \mathrm{SA}$, $\mathrm{C}_{17} \mathrm{SO}, \mathrm{C}_{17} \mathrm{SA}, \mathrm{C}_{18} \mathrm{SO}, \mathrm{C}_{18} \mathrm{SA}, \mathrm{C}_{20} \mathrm{SO}, \mathrm{C}_{18} \mathrm{SA}$-diene, doxSA and doxSO.

\section{Statistics}

Mean comparison and receiver operating characteristic curve analyses The original dataset was imported into SPSS 16.0 (SPSS Switzerland, Zurich, Switzerland). As some of the variables measured did not follow normal distribution, even after transformations, non-parametric tests were used. The Kruskal-Wallis test was used to compare all three groups. The Mann-Whitney $U$ test was used for comparisons between two groups, followed by the Bonferroni correction. Spearman correlations were calculated. Receiver operating characteristic (ROC) curve analysis was performed and the two-tailed asymptotic $p$ value was calculated for the ROC AUCs.

Orthogonal partial least square-discriminant analysis Orthogonal partial least square-discriminant analysis (OPLSDA) $[22,23]$ was used as a multivariate predictive and regression method, based on the concepts of principal component analysis (PCA). In PCA, a large number of correlated variables (e.g. clinical, clinical chemistry and sphingolipid variables) are summarised into a smaller number of uncorrelated ones called principal components. 
Thus, the correlation structures are preserved while reducing the number of variables. In contrast to PCA, which does not consider class membership, in OPLS-DA the group information is included (control, the non-diabetic metabolic syndrome and diabetes) as a $Y$ variable. OPLS-DA therefore summarises the data into a predictive component, maximising the between-group variation and an orthogonal component(s) describing the within-group variation. The predictive component describes the variations in $X$ that correlate with $Y$. The orthogonal component describes the systematic variations within each group that do not correlate with $Y$, thereby removing the noise data from the predictive component.

For any modelling technique, model evaluation is necessary before any inferences are made. $R^{2} Y$ reports the fit of the model to the original dataset and $Q^{2} Y$ reports the predictive ability of the model calculated by cross validation. Cross validation is performed by dividing the whole dataset into multiple random groups and then predicting the class assignment of members of each group at a time.

The dataset was imported into SIMCA-P +12.0.1.0 (Umetrics, Umeå, Sweden) for the calculations. The groups (control, non-diabetic metabolic syndrome and diabetes) were assigned as classes. Variables that did not follow the normal distribution were $\log$ transformed before model fitting. As some variables did not follow the normal distribution after log transformation, models were fitted twice, once with the log-transformed variables and another time without any transformations. The model evaluation parameters and interpretations did not differ in both cases. Therefore, the values for all the variables without any transformations were used. As the values for the variables had different scales, the dataset was normalised in unit variance and centred around the mean. OPLS-DA models were fitted for the classes to get the highest $R^{2} Y$ and $Q^{2} Y$ values. Models were used to compare two groups only at a time (control vs non-diabetic metabolic syndrome and nondiabetic metabolic syndrome vs diabetes). We did not use three groups as a $Y$ variable as this would have made the interpretations of the model results more complex.

As PCA-based methods are sensitive to outliers, Hotelling's $T^{2}$ and distance to the model, DmodX, were used to detect outliers. Hotelling's $T^{2}$ is a generalisation of the $t$ distribution for the multivariate case and is usually visualised in score plots. Score plots show on the $x$-axis the scores of the predictive component describing the between-group variation and on the $y$-axis the scores of the orthogonal component describing the within-group variation. Tolerance ellipse is drawn in score plots outlining the $95 \%$ probability of the Hotelling's $T^{2}$ distribution. Scores for observations situated outside the tolerance ellipse can be considered outliers. Moreover, DModX shows the distance to the model in the $X$ space in a way similar to the residuals in the linear regression models. A critical value D-Crit of 0.05 was set as a limit for outlier detection. After outliers were detected, models were fitted twice, excluding and including the outliers. In the current study, no difference was found in the model evaluation parameters or any of the results upon inclusion or exclusion of the outliers. Therefore, models without outlier removal were used for the interpretation.

Cross validation was performed for each model with seven groups. The cross validation ANOVA was calculated along with misclassification tables and Fisher's probabilities.

\section{Results}

For this study, we analysed plasma samples from patients in three pre-defined subgroups, each consisting of 25 sexand age-matched patients with either manifest type 2 diabetes mellitus, the metabolic syndrome but no manifest diabetes mellitus, or without diabetes mellitus or the metabolic syndrome (controls). The results are summarised in Table 1.

Sphingoid bases in plasma are usually $N$-acylated and conjugated with different headgroups, giving rise to a great variety of different sphingolipid species. To analyse the sphingoid base composition of these metabolites we subjected the extracted lipids to sequential acid and base hydrolysis to remove the $N$-acyl chain and head group. The resulting free sphingoid bases were analysed by LC/MS.

The most abundant sphingoid base in human plasma was $\mathrm{C}_{18}$-sphingosine $\left(\mathrm{C}_{18} \mathrm{SO}\right)$ followed by $\mathrm{C}_{18} \mathrm{SA}$-diene, $\mathrm{C}_{16}$-sphingosine $\left(\mathrm{C}_{16} \mathrm{SO}\right)$ and $\mathrm{C}_{17}$-sphingosine $\left(\mathrm{C}_{17} \mathrm{SO}\right)$. Generally, dSLs were recovered as quantitatively minor fractions, representing $0.1-0.3 \%$ of the total sphingoid bases

Plasma concentrations of total dSLs were significantly higher for the non-diabetic metabolic syndrome group $(0.11 \pm 0.04 \mu \mathrm{mol} / \mathrm{l})$ compared with controls $(0.06 \pm 0.02 \mu \mathrm{mol} / \mathrm{l})$ but did not differ between the metabolic syndrome and diabetic groups (Table 1). This elevation remained significant after the Bonferroni correction for multiple testing. In contrast, $\mathrm{C}_{16} \mathrm{SO}$ levels were found to be significantly lower $(p=0.008)$ in diabetic patients $(6.37 \pm 2.82 \mu \mathrm{mol} / \mathrm{l})$ in comparison with participants with non-diabetic metabolic syndrome (8.38 \pm $2.25 \mu \mathrm{mol} / \mathrm{l})$. However, this decrease missed the significance limit after Bonferroni correction in which a significance level of 0.05 corresponds to a $p$ value of 0.002. Concentrations of other sphingoid bases were not significantly different between the three groups. 
Table 2 Results of STZ rat tissue and plasma analysis

Values are shown as mean $\pm \mathrm{SD}$

$p$ values were calculated with Mann-Whitney $U$ test: $* p<0.05 ; * * * p<0.001$

$C$, controls; ND, not detected; STZ, STZ-treated rats

\begin{tabular}{|c|c|c|c|c|c|c|}
\hline \multirow[t]{2}{*}{ Variable } & \multicolumn{2}{|c|}{ Liver (pmol/100 $\mu$ g protein) } & \multicolumn{2}{|c|}{$\begin{array}{l}\text { Muscle }(\mathrm{pmol} / 100 \mu \mathrm{g} \\
\text { protein) }\end{array}$} & \multicolumn{2}{|c|}{ Plasma $(\mu \mathrm{mol} / \mathrm{l})$} \\
\hline & $\mathrm{C}$ & STZ & $\mathrm{C}$ & STZ & $\mathrm{C}$ & STZ \\
\hline $\begin{array}{l}\text { Glucose } \\
(\mathrm{mmol} / \mathrm{l})\end{array}$ & - & - & - & - & $8.8 \pm 0.4$ & $53.8 \pm 3.3 * * *$ \\
\hline $\mathrm{C}_{16} \mathrm{SO}$ & $3.4 \pm 0.8$ & $3.1 \pm 0.7$ & $1.5 \pm 0.23$ & $1.4 \pm 0.24$ & $0.06 \pm 0.02$ & $0.09 \pm 0.07$ \\
\hline $\mathrm{C}_{17} \mathrm{SO}$ & $15.7 \pm 3.3$ & $13 \pm 2.5$ & $4.2 \pm 0.44$ & $5.0 \pm 1.2$ & $0.3 \pm 0.1$ & $0.4 \pm 0.1$ \\
\hline $\mathrm{C}_{18} \mathrm{SA}$-diene & $64.1 \pm 10.6$ & $78.1 \pm 26.1$ & $11.2 \pm 2.3$ & $21.5 \pm 10.3^{*}$ & $2.6 \pm 0.4$ & $5.0 \pm 0.9 *$ \\
\hline $\mathrm{SO}$ & $1,130 \pm 189$ & $1,127 \pm 252$ & $180 \pm 25.4$ & $262 \pm 106^{*}$ & $24.3 \pm 7.6$ & $29.1 \pm 4.8$ \\
\hline SA & $40.3 \pm 9.4$ & $66.4 \pm 29 *$ & $9.1 \pm 1.8$ & $8.1 \pm 2.6$ & $1.0 \pm 0.5$ & $1.6 \pm 0.6^{*}$ \\
\hline $\mathrm{C}_{20} \mathrm{SO}$ & $10.8 \pm 4.4$ & $11.4 \pm 2$ & $6.5 \pm 0.6$ & $6.7 \pm 1.6$ & $0.1 \pm 0.08$ & $0.15 \pm 0.9$ \\
\hline DoxSA & $0.2 \pm 0.08$ & $0.4 \pm 0.12 * * *$ & ND & ND & $0.01 \pm 0.003$ & $0.12 \pm 0.9 * * *$ \\
\hline DoxSO & ND & ND & ND & ND & ND & ND \\
\hline
\end{tabular}

A correlation matrix of all variables (clinical data, clinical chemistry measurements along with sphingoid base backbone measurements) showed a significant correlation of the serine-based sphingolipids $\left(\mathrm{C}_{16}, \mathrm{C}_{17}\right.$ or $\mathrm{C}_{18} \mathrm{SA}$ or $\mathrm{SO}$ and $\mathrm{C}_{18} \mathrm{SA}$-diene) with LDL-cholesterol and total cholesterol but correlated less strongly with HDL-cholesterol. In contrast, the alanine-based dSLs showed a strong correlation with the variables related to the metabolic syndrome, such as waist circumference and triacylglycerols (ESM Fig. 2). Therefore, we were interested to see whether the increased dSL levels are associated with hyperglycaemia per se or rather with insulin resistance. To address this issue we analysed plasma, liver and muscle tissue samples from STZ-treated rats (Table 2). Although beta cell failure and not insulin resistance is the basis of the STZ rat model, we found significantly elevated dSL levels in plasma and liver from STZ rats (Table 2, Fig. 1). No dSLs were detected in muscle tissue. This suggests that plasma dSLs are primarily elevated in association with hyperglycaemia and are probably of hepatic origin.

An OPLS-DA was used to estimate the importance of the individual variables as discriminating biomarkers. Both models (control vs non-diabetic metabolic syndrome and non-diabetic metabolic syndrome vs diabetes) showed good fit and predictive power. Therefore, they are valid for discrimination (ESM Table 1).

In score plots (Fig. 2a,b), clustering into separate groups was preserved after reducing the whole dataset into a single predictive component (shown on the $x$-axis) and an orthogonal one (shown on the $y$-axis). Loading plots (Fig. 2c,d) show the weights of each of the original variables in the model and hence their individual contributions to the disease state. In the control vs metabolic syndrome loading plot (Fig. 2c), triacylglycerols, doxSA, doxSO, and diastolic and systolic blood pressure contributed to the metabolic syndrome state model $(95 \% \mathrm{CI}>0)$ whereas elevated HDL-cholesterol contributed to the control state model $(95 \% \mathrm{CI}<0)$. This confirms the biological and clinical validity of the model, as elevated triacylglycerols, low HDL-cholesterol and hypertension are key features of the metabolic syndrome. DoxSA and doxSO were identified as the second and fourth most important contributors to the metabolic syndrome model, just after triacylglycerols and HDL-cholesterol, respectively, but above glucose, waist circumference and systolic blood pressure. In the metabolic syndrome vs diabetes
Fig. 1 DoxSA levels are significantly $(p<0.001)$ elevated in liver tissue (a) and plasma (b) of STZ rats (STZ) compared with controls $(\mathrm{C})$
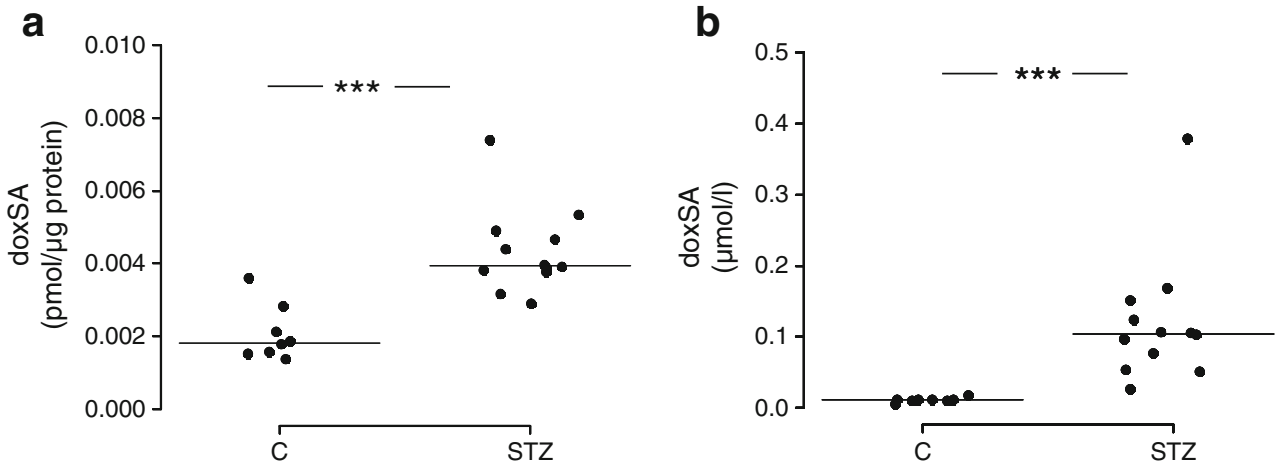
a
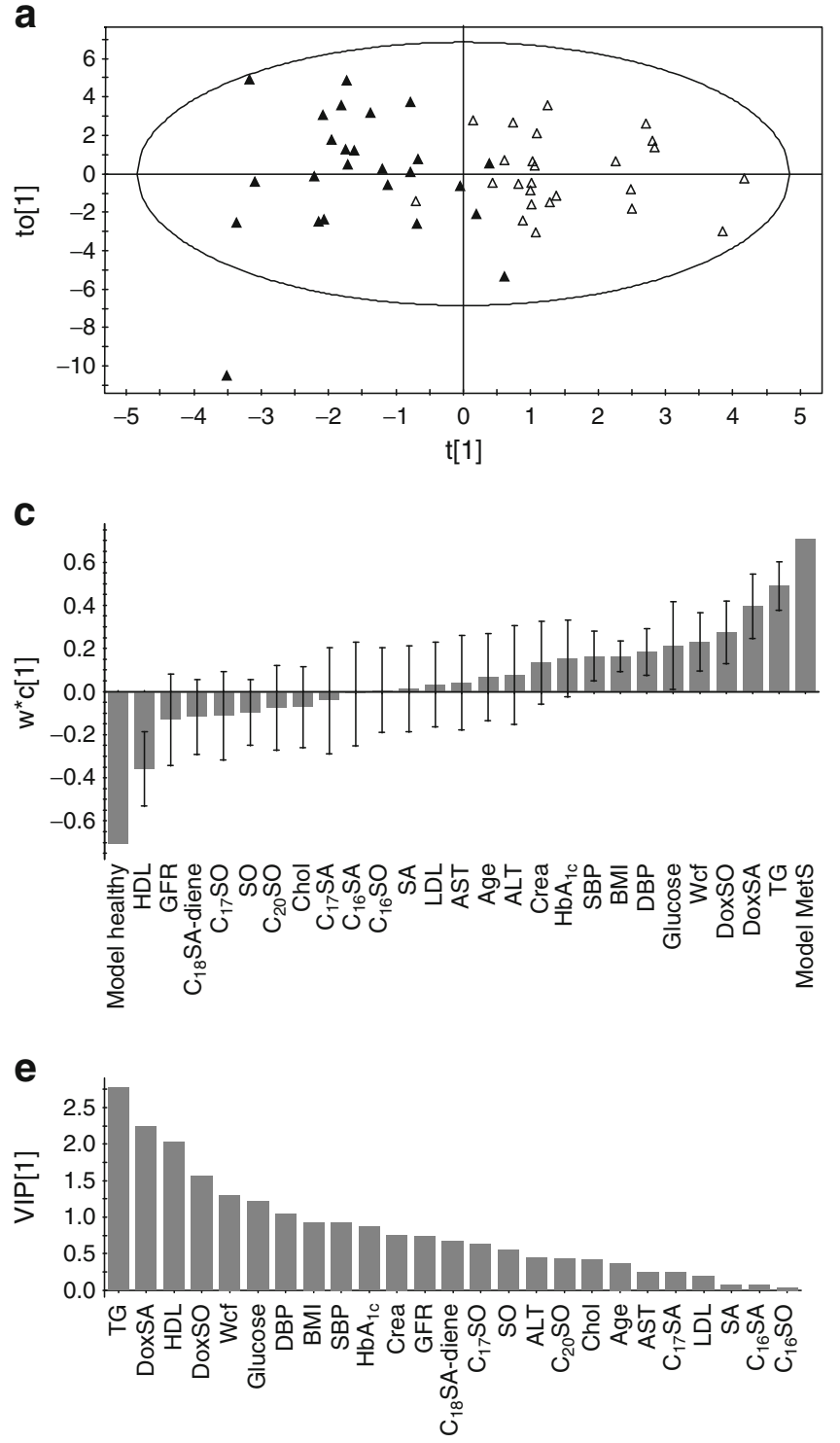

Fig. 2 a,b Score plots of OPLS-DA models: (a) control vs non-diabetic metabolic syndrome; (b) non-diabetic metabolic syndrome vs type 2 diabetes. Individual observations are shown as: black triangles, controls; white triangles, the metabolic syndrome; black rhombus, type 2 diabetes. On the $x$-axis, the scores for each individual in the predictive principal component (t[1]) are shown, while the $y$-axis shows the scores for each individual in the orthogonal component (to[1]). Thus, variations on the $x$-axis reflect the between-group separation while variations on the $y$-axis show the within-group variations (noise). The tolerance ellipse corresponds to $95 \%$ of the Hotelling's $T^{2}$ multivariate distribution. c,d Loading column plots of OPLS-DA models control vs the metabolic syndrome (c) and the metabolic syndrome vs diabetes (d). The variables are shown on the $x$-axis, while the loading coefficients

model (Fig. 2d), we found elevated fasting glucose and $\mathrm{HbA}_{1 \mathrm{c}}$ concentrations, together with low $\mathrm{C}_{16} \mathrm{SO}$ and creatinine levels, to be the contributors to the diabetes state model.

Variable importance for the projection (VIP) plots (Fig. 2e,f) show the contribution of each variable to the
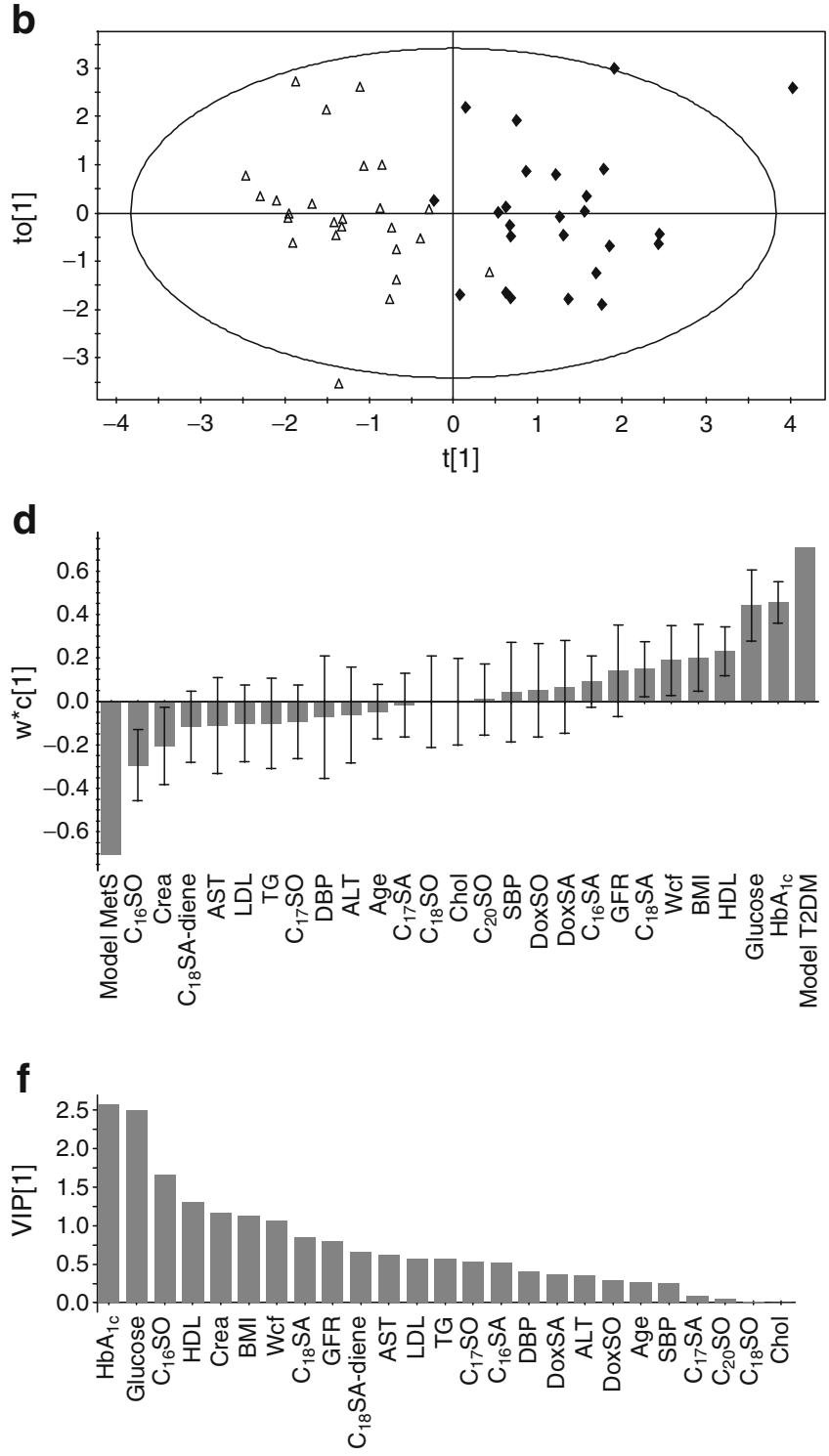

(weights) are shown on the $y$-axis. The weights represent the contribution of each variable to the model component scores. Variables with larger weights contribute more to the model. Error bars represent 95\% CIs for calculated weights. e,f VIP plot for OPLS-DA models: control vs the metabolic syndrome (e) and the metabolic syndrome vs diabetes (f). The variables are shown on the $x$-axis, while VIP coefficients are shown on the $y$-axis. The VIP coefficients plot shows the summation of all the weights for each $X$ variable to predict $Y$ and hence denoting the importance of each $X$ variable. AST, aspartate aminotransferase; Chol, cholesterol; Crea, creatinine; DBP, diastolic BP; HDL, HDL-cholesterol; LDL, LDL-cholesterol; SBP, systolic BP; T2DM, type 2 diabetes mellitus; TG, triacylglycerol; Wcf, waist circumference

variation in both the $X$ space and the $Y$ space (and hence their correlations with other variables and the control or disease state). A coefficient value $>1$ signifies that the variable is important. For the control vs metabolic syndrome model (Fig. 2e), the VIP plot showed the 
highest VIP coefficients for triacylglycerols, doxSA, HDL-cholesterol and doxSO. A slight importance (VIP coefficient slightly $>1$ ) was noticed for diastolic pressure and fasting glucose. For the metabolic syndrome vs type 2 diabetes mellitus model (Fig. 2f) a significant importance of $\mathrm{HbA}_{1 \mathrm{c}}$, glucose and $\mathrm{C}_{16} \mathrm{SO}$ was seen. VIP coefficients were also $>1$ for BMI, HDL-cholesterol and creatinine.

In summary, the OPLS-DA analysis revealed that triacylglycerols, doxSA, doxSO and HDL-cholesterol are the best explanatory variables for the non-diabetic metabolic syndrome, while differences in $\mathrm{HbA}_{1 \mathrm{c}}$, glucose and $\mathrm{C}_{16} \mathrm{SO}$ were mostly related to the diabetes.

The diagnostic potential of these markers was analysed in an ROC curve analysis (Fig. 3a,b). For the diagnosis of the metabolic syndrome, doxSA and doxSO (Fig. 3a) had AUCs of 0.875 and 0.842 , respectively $(p<0.001)$. Moreover, $\mathrm{C}_{16} \mathrm{SO}$ (Fig. 3b) showed an AUC of 0.282 (corresponding to $0.718 ; p<0.01$ ).

\section{Discussion}

In this study, we compared the plasma sphingoid base compositions of healthy individuals with those of individuals with non-diabetic metabolic syndrome and diabetic patients. We found dSLs to be significantly elevated in the plasma of patients with either non-diabetic metabolic syndrome or type 2 diabetes mellitus compared with controls but not different between participants with nondiabetic metabolic syndrome and diabetic patients. In contrast, $\mathrm{C}_{16} \mathrm{SO}$ levels were lower in diabetic patients but did not differ between controls and metabolic syndrome patients. Other sphingoid base metabolites were not different between the groups (Table 1).

These results indicate that the metabolic changes in the metabolic syndrome and type 2 diabetes mellitus are specifically associated with alterations in some, but not all, sphingoid base species. Elevated dSL levels were also confirmed in the plasma and liver tissue of a model of type 1 diabetes (STZ rats), indicating that the observed increase in dSLs is independent of the type of diabetes.

Sphingolipid metabolism can be considered as a metabolic cross point that interconnects fatty acid (acyl-CoA) and amino acid (serine and alanine) metabolism. Serine and alanine formation is thereby linked to glycolysis, which forms their precursors, 3-phosphoglycerate and pyruvate, respectively.

The metabolic syndrome and type 2 diabetes mellitus are associated with the clustering of several risk factors. To delineate the relative contribution of each variable we used a supervised learning approach (OPLS-DA) [24, 25]. This analysis showed that dSL levels have, in addition to triacylglycerols and HDL-cholesterol, the highest explana-
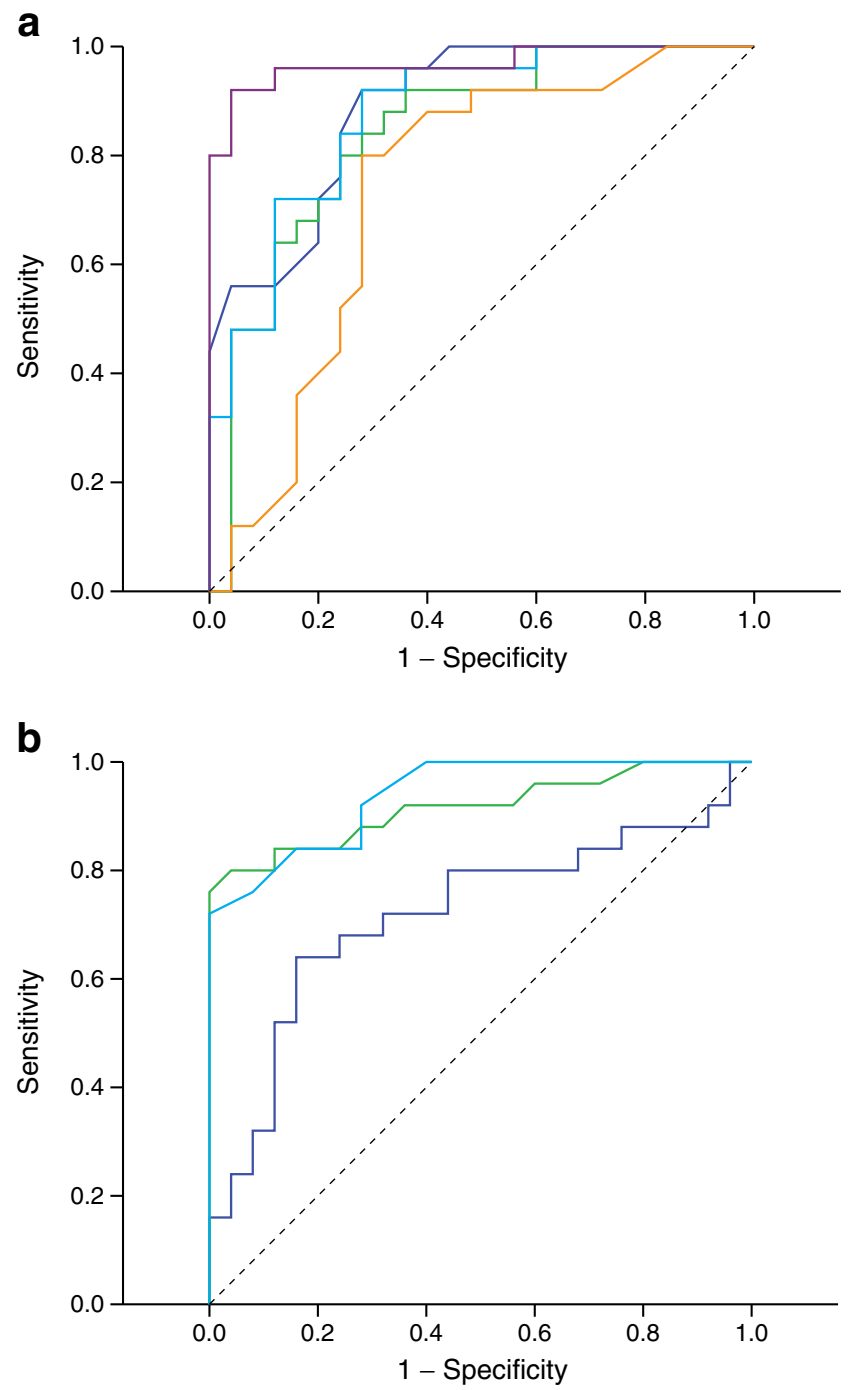

Fig. 3 ROC curves. (a) ROC curves for control vs metabolic syndrome showing triacylglycerols and HDL-cholesterol as predictors of the metabolic syndrome with AUCs of $0.968(p<0.001)$ and 0.111 (corresponding to 0.889 ), respectively. The dSLs show comparable AUCs, with 0.875 for doxSA and 0.842 for doxSO. Purple, triacylglycerol; dark blue, HDL-cholesterol (reciprocal); green, doxSO; light blue, doxSA; orange, waist circumference. (b) ROC curves for potential biomarkers in metabolic syndrome vs diabetes. $\mathrm{HbA}_{1 \mathrm{c}}$ and glucose show AUCs of 0.939 and 0.917 for type 2 diabetes, whereas $\mathrm{C}_{16} \mathrm{SO}$ shows a significantly lower AUC of 0.282 (corresponding to 0.718 ), denoting its decrement in association with diabetes. Green, glucose; light blue, $\mathrm{HbA}_{1 \mathrm{c}}$; dark blue, $\mathrm{C}_{16} \mathrm{SO}$ (reciprocal)

tory power for the metabolic syndrome. In this context it has to be considered that the metabolic syndrome and type 2 diabetes mellitus are clinically not two completely separate entities. Most diabetic patients also fulfil the criteria for the metabolic syndrome which, by itself, is associated with insulin resistance. In fact, most diabetic patients have presented for many years with the metabolic syndrome before hyperglycaemia has manifest. The observation that dSL levels are 
not different between non-diabetic metabolic syndrome patients and diabetic patients suggests that the dSLs are already formed in the prediabetic state in which insulin insensitivity is still compensated by increased insulin production and hyperinsulinaemia.

In contrast, $\mathrm{C}_{16} \mathrm{SO}$ together with glucose and $\mathrm{HbA}_{1 \mathrm{c}}$ were the strongest contributors in the diabetes model. $\mathrm{C}_{16} \mathrm{SO}$ is generated by the use of myristoyl-CoA instead of palmitoyl $\mathrm{CoA}-\mathrm{a}$ reaction that is primarily catalysed by the SPT long-chain base subunit 3 (SPTLC3) [13].

It should be noted at this point that we also found significant levels of $\mathrm{C}_{17} \mathrm{SO}$ in the plasma samples analysed. $\mathrm{C}_{17} \mathrm{SO}$ is considered to be an 'unnatural' sphingoid base and therefore is sometimes used as an internal normalisation standard in lipidomics. However, the identity of $\mathrm{C}_{17} \mathrm{SO}$ in human plasma was validated by comparison with a commercial synthetic $\mathrm{C}_{17} \mathrm{SO}$ standard and also reported earlier by Quehenberger et al. [26]. This indicates that $\mathrm{C}_{17} \mathrm{SO}$ has to be used with caution as an internal standard for plasma samples.

Currently, we do not fully understand why plasma dSL levels are increased in the metabolic syndrome and diabetes. That dSLs are almost exclusively present in VLDL and LDL but not HDL indicates that the dSLs in plasma are primarily of hepatic origin [27]. This view is supported by the observation that dSL levels were elevated in liver and plasma of STZ rats but were not present in skeletal muscle. A possible explanation for the increased dSL formation might be an increased hepatic availability of alanine. Recent reports show that lifestyle modifications in the metabolic syndrome are associated with significant changes in the plasma amino acid profiles [28]. In obese individuals increases in skeletal muscle output of alanine and hepatic uptake of alanine were seen while serine levels were not changed [18]. Furthermore, hepatic glucose uptake is primarily mediated by GLUT2 and hence is insulin independent. Hyperglycaemic conditions are therefore associated with elevated hepatic glucose levels and an increased glycolytic flux that increases the formation of pyruvate and its anaerobic conversion to either lactate or alanine. Elevated glucose levels could hence increase hepatic alanine levels and thereby dSL generation. This model implies that dSL levels are elevated independent of the type of diabetes, which is supported by the observation that dSL levels were also found to be elevated in the plasma and liver of an animal model of type 1 diabetes (STZ rats). In both conditions even short-term and transient fluctuations in plasma glucose levels might be integrated over time leading to increased formation of dSLs that, by themselves, might have a slow turnover as they are not degraded by the canonical sphingolipid catabolism.

Another interesting aspect is whether increased plasma dSL levels as well as decreased plasma $\mathrm{C}_{16} \mathrm{SO}$ levels contribute to the pathogenesis of diabetes and its complications. Genome-wide association studies have shown a strong association of genetic SPTLC3 variants with alterations in lipid metabolism [29] and increased risk for myocardial infarction [30]. Therefore, lower $\mathrm{C}_{16} \mathrm{SO}$ levels might be directly or indirectly related to the increased risk of diabetic patients for developing cardiovascular complications.

Previously, we showed that the increased formation of dSLs is the pathological cause of the inherited neuropathy HSAN1 $[15,31]$. Clinically, HSAN1 closely resembles the diabetic peripheral neuropathy (DPN) that occurs in about $60 \%$ of diabetic patients. Both conditions have late onset, slow progression and length-dependent axonopathy. All peripheral nerves are affected, including pain fibre, motor neurons and autonomic nerves. The degeneration of small sensory fibres results in the loss of pain sensation, which in turn leads to painless injuries. Furthermore, HSAN1 and DPN are both associated with skin ulcers, which is not a common feature in other peripheral neuropathies. Considering the neurotoxic properties of dSLs, it might therefore be conceivable that higher levels of dSLs are not only interesting from the biomarker perspective but also because dSLs are potential pathogenic agents in the DPN. Interestingly, it has been shown recently that serum triacylglycerols correlate with the progression of the diabetic neuropathy [32]. In parallel we observed a strong correlation of the dSLs and triacylglycerol levels (ESM Fig. 2).

Taken together our results suggest that dSLs are relevant biomarkers for both the metabolic syndrome and type 2 diabetes mellitus, whereas $\mathrm{C}_{16} \mathrm{SO}$ bases seem to be specifically lowered in diabetes, possibly reflecting the risk for developing cardiovascular complications. Therefore, a combination of these markers might help to improve risk prediction and therapy monitoring in diabetic patients. This could be especially relevant in the transition from the prediabetic to the diabetic state. A limitation of this study is the rather small group size. However, for a pilot biomarker study it is valid to use small numbers as long as type I and type II errors are considered carefully. We showed $p$ values in the order of $1 \times 10^{-5}$ after the most conservative correction for doxSA and doxSO, which support rejection of the null hypothesis, that there is no difference in their plasma levels between the different groups. However, larger and ideally prospective follow-up studies are needed to further validate the potential of these metabolites and to explore their diagnostic and prognostic value. Finally, a more detailed knowledge of the physiological and pathophysiological properties of these metabolites is important to better understand the interplay between sphingolipids, carbohydrate metabolism, insulin resistance and diabetes. 
Acknowledgements The work was undertaken at the University Hospital of Zurich and was supported by grants from the Hartmann Müller Foundation, the Herzog-Egli Foundation, the Olga Mayenfisch Foundation and the Foundation for Scientific Research (University of Zurich) as well as the German Society for Clinical Chemistry and Laboratory Medicine (DGKL), the Gebert Rüf Foundation, Centre for Integrative Human Physiology (ZIHP, University of Zurich) and the European Commission (LSHM-CT-2006-037631).

Contribution statement AO, MFR and DE did the lipid extraction and analysis, CHS, PR and HD were involved in clinical study design, sample collection and patient characterisation, CP-S, GLP, RB conceived and designed the rat experiments. AO did the statistical analysis, AvE contributed to study design, data interpretation and $\mathrm{TH}$ was involved in study design, data interpretation and wrote the manuscript. All authors had a substantial input in drafting the manuscript, critically appraising it and approved the final version to be published.

Duality of interest The authors declare that there is no duality of interest associated with this manuscript.

\section{Appendix}

\section{Chemical nomenclature}

$\mathrm{C}_{16} \mathrm{SA}$ or $\mathrm{C}_{16}$-D-erythro-
sphinganine
$\mathrm{C}_{17} \mathrm{SA}$
$\mathrm{C}_{18} \mathrm{SA}$
$\mathrm{C}_{20} \mathrm{SA}$
$\mathrm{C}_{16} \mathrm{SO}$ or $\mathrm{C}_{16}$ D-erythro-
sphingosine
$\mathrm{C}_{17} \mathrm{SO}$
$\mathrm{C}_{18} \mathrm{SO}$
$\mathrm{C}_{20} \mathrm{SO}$

$(2 S, 3 R, 4 E)-2-$

Aminohexadecan-1,3-diol

$(2 S, 3 R, 4 E)-2-$

Aminoheptadecan-1,3-diol

$(2 S, 3 R, 4 E)-2-$

Aminooctadecan-1,3-diol

$(2 S, 3 \mathrm{R}, 4 E)-2$-Aminoicosan-

1,3-diol

$(2 S, 3 R, 4 E)$-2-Amino-4-

hexadecen-1,3-diol

$(2 S, 3 R, 4 E)$-2-Amino-4-

heptadecen-1,3-diol

$(2 S, 3 R, 4 E)$-2-Amino-4-

octadecen-1,3-diol

$(2 S, 3 R, 4 E)$-2-Amino-4-icosen-

1,3-diol

\section{References}

1. IUPAC-IUB Commission on Biochemical Nomenclature (1978) The nomenclature of lipids (Recommendations 1976) IUPAC-IUB Commission on Biochemical Nomenclature. Biochem J 171:21-35

2. Donath MY, Halban PA (2004) Decreased beta-cell mass in diabetes: significance, mechanisms and therapeutic implications. Diabetologia 47:581-589

3. Kahn SE, Hull RL, Utzschneider KM (2006) Mechanisms linking obesity to insulin resistance and type 2 diabetes. Nature 444:840-846

4. Prentki M, Nolan CJ (2006) Islet beta cell failure in type 2 diabetes. J Clin Invest 116:1802-1812

5. DeFronzo RA (2010) Insulin resistance, lipotoxicity, type 2 diabetes and atherosclerosis: the missing links. The Claude Bernard Lecture 2009. Diabetologia 53:1270-1287

6. McGarry JD (1992) What if Minkowski had been ageusic? An alternative angle on diabetes. Science 258:766-770

7. Chavez JA, Summers SA (2010) Lipid oversupply, selective insulin resistance, and lipotoxicity: molecular mechanisms. Biochim Biophys Acta 1801:252-265

8. Olefsky JM, Glass CK (2010) Macrophages, inflammation, and insulin resistance. Annu Rev Physiol 72:219-246

9. Cowart LA (2009) Sphingolipids: players in the pathology of metabolic disease. Trends Endocrinol Metab 20:34-42

10. Summers SA (2006) Ceramides in insulin resistance and lipotoxicity. Prog Lipid Res 45:42-72

11. Deevska GM, Nikolova-Karakashian MN (2011) The twists and turns of sphingolipid pathway in glucose regulation. Biochimie 93:32-38

12. Holland WL, Brozinick JT, Wang LP et al (2007) Inhibition of ceramide synthesis ameliorates glucocorticoid-, saturated-fat-, and obesity-induced insulin resistance. Cell Metab 5:167-179

13. Hornemann T, Penno A, Rutti MF et al (2009) The SPTLC3 subunit of serine palmitoyltransferase generates short chain sphingoid bases. J Biol Chem 284:26322-26330

14. Zitomer NC, Mitchell T, Voss KA et al (2009) Ceramide synthase inhibition by fumonisin B1 causes accumulation of 1-deoxysphinganine: a novel category of bioactive 1-deoxy-sphingoid bases and 1-deoxy-dihydroceramides biosynthesized by mammalian cell lines and animals. J Biol Chem 284:4786-4795

15. Penno A, Reilly MM, Houlden H et al (2010) Hereditary sensory neuropathy type 1 is caused by the accumulation of two neurotoxic sphingolipids. J Biol Chem 285:11178-11187

16. Humpf HU, Schmelz EM, Meredith FI et al (1998) Acylation of naturally occurring and synthetic 1-deoxysphinganines by ceramide synthase. Formation of N-palmitoyl-aminopentol produces a toxic metabolite of hydrolyzed fumonisin, AP1, and a new category of ceramide synthase inhibitor. J Biol Chem 273:19060-19064

17. Hu W, Bielawski J, Samad F, Merrill AH Jr, Cowart LA (2009) Palmitate increases sphingosine-1-phosphate in $\mathrm{C} 2 \mathrm{C} 12$ myotubes via upregulation of sphingosine kinase message and activity. J Lipid Res 50:1852-1862

18. Felig P, Wahren J, Hendler R, Brundin T (1974) Splanchnic glucose and amino acid metabolism in obesity. J Clin Invest 53:582-590

19. Wahren J, Felig P, Cerasi E, Luft R (1972) Splanchnic and peripheral glucose and amino acid metabolism in diabetes mellitus. J Clin Invest 51:1870-1878

20. Saely CH, Vonbank A, Rein P et al (2008) Alanine aminotransferase and gamma-glutamyl transferase are associated with the metabolic syndrome but not with angiographically determined coronary atherosclerosis. Clin Chim Acta 397:82-86

21. Riley RT, Norred WP, Wang E, Merrill AH (1999) Alteration in sphingolipid metabolism: bioassays for fumonisin- and ISP-I-like activity in tissues, cells and other matrices. Nat Toxins 7:407-414

22. Trygg J, Wold S (2002) Orthogonal projections to latent structures (O-PLS). J Chemometr 16:119-128

23. Bylesjö M, Rantalainen M, Cloarec O, Nicholson JK, Holmes E, Trygg J (2006) OPLS discriminant analysis: combining the strengths of PLS-DA and SIMCA classification. J Chemometr 20:341-351

24. Tsutsui H, Maeda T, Toyo'oka T et al (2010) Practical analytical approach for the identification of biomarker candidates in prediabetic state based upon metabonomic study by ultraperformance liquid chromatography coupled to electrospray ionization time-of-flight mass spectrometry. J Proteome Res 9:3912-3922

25. Qiu Y, Cai G, Su M et al (2010) Urinary metabonomic study on colorectal cancer. J Proteome Res 9:1627-1634 
26. Quehenberger O, Armando AM, Brown AH et al (2010) Lipidomics reveals a remarkable diversity of lipids in human plasma. J Lipid Res 51:3299-3305

27. Bertea M, Rutti MF, Othman A et al (2010) Deoxysphingoid bases as plasma markers in diabetes mellitus. Lipids Health Dis 9:84

28. Kamaura M, Nishijima K, Takahashi M, Ando T, Mizushima S, Tochikubo O (2010) Lifestyle modification in metabolic syndrome and associated changes in plasma amino acid profiles. Circ J 74:2434-2440

29. Illig T, Gieger C, Zhai G et al (2010) A genome-wide perspective of genetic variation in human metabolism. Nat Genet 42:137-141
30. Hicks AA, Pramstaller PP, Johansson A et al (2009) Genetic determinants of circulating sphingolipid concentrations in European populations. PLoS Genet 5:e1000672

31. Eichler FS, Hornemann T, McCampbell A et al (2009) Overexpression of the wild-type SPT1 subunit lowers desoxysphingolipid levels and rescues the phenotype of HSAN1. J Neurosci 29:14646-14651

32. Wiggin TD, Sullivan KA, Pop-Busui R, Amato A, Sima AA, Feldman EL (2009) Elevated triglycerides correlate with progression of diabetic neuropathy. Diabetes 58:1634-1640 